\title{
Belgeo
}

Revue belge de géographie

\section{Application de méthodes de prospection à l'étude géo-archéologique de la plaine côtière du fleuve Potenza (Les Marches, Italie)}

Application of prospecting methods in the geo-archaeological study of the coastal plain of the Potenza river (The Marches, Italy)

Tanja Goethals, Morgan De Dapper, Frank Vermeulen, Dirk Van Damme et Kristine Walraevens

\section{(2) OpenEdition}

\section{Journals}

Édition électronique

URL : http://journals.openedition.org/belgeo/11959

DOI : 10.4000/belgeo.11959

ISSN : 2294-9135

Éditeur :

National Committee of Geography of Belgium, Société Royale Belge de Géographie

Édition imprimée

Date de publication : 30 septembre 2006

Pagination : 339-362

ISSN : 1377-2368

Référence électronique

Tanja Goethals, Morgan De Dapper, Frank Vermeulen, Dirk Van Damme et Kristine Walraevens, « Application de méthodes de prospection à l'étude géo-archéologique de la plaine côtière du fleuve Potenza (Les Marches, Italie) », Belgeo [En ligne], 3 | 2006, mis en ligne le 30 octobre 2013, consulté le 01 mai 2019. URL : http://journals.openedition.org/belgeo/11959; DOI : 10.4000/belgeo.11959

Ce document a été généré automatiquement le 1 mai 2019.

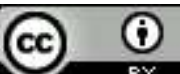

Belgeo est mis à disposition selon les termes de la licence Creative Commons Attribution 4.0 International. 


\section{Application de méthodes de prospection à l'étude géo- archéologique de la plaine côtière du fleuve Potenza (Les Marches, Italie)}

Application of prospecting methods in the geo-archaeological study of the coastal plain of the Potenza river (The Marches, Italy)

Tanja Goethals, Morgan De Dapper, Frank Vermeulen, Dirk Van Damme et Kristine Walraevens

Ce projet de recherche a bénéficié du soutien financier du Fonds Spécial pour la Recherche de l'Université de Gand, du Fonds pour la Recherche Scientifique de la Région Flamande, et du Département de la Politique Scientifique Fédérale de la Belgique (projet IPA/5). Le projet a largement profité de la collaboration étroite avec diverses institutions italiennes que nous tenons à remercier : la Soprintendenza per i Beni Archeologici delle Marche, l'Ufficio Cartografico de la Regione Marche, les Universités de Camerino et de Macerata, le Service de Pédologie de l'ASSAM, le Département de Géologie de l'Université de Siena et la Faculté d'Agronomie de l'Université de Florence. Nous remercions également tous les relecteurs et les rédacteurs pour leurs suggestions et critiques qui ont contribué à améliorer le manuscrit.

\section{Abridged English version}

1 This contribution focuses on geo-archaeological methodology as applied to the coastal plain of the Potenza river valley. This $80 \mathrm{~km}$-long and $10 \mathrm{~km}$-wide WNW-ENE-trending fluvial basin (fig. 1 ) is the subject of study of the Potenza Valley Survey project (University of Ghent, Belgium) since 2000. The principal aims of this multidisciplinary project are the reconstruction of the evolution of 
human occupation and landscape and their interaction between 1000 BC and $1000 \mathrm{AD}$; and the refining of geo-archaeological methodology.

The $4 \mathrm{~km}$-wide coastal plain of the river Potenza is flanked on the northern and southern sides by hills reaching their maximum at $+150 \mathrm{~m}$ to $+200 \mathrm{~m}$ elevation, which end at the eastern side in abandoned cliffs (fig. 2). They consist of Early Pleistocene clays and sands covered by a marine terrace. The plain is filled with Quaternary and Holocene sediments down to a depth of at least 50 $m$. After the last glacial erosion phase, the Flandrian transgression pushed the coastline inland, forming an Atlantic-type coast of alternating cliffs and bays. With the slowing of the sea-level rise, compound spits developed into beach ridges. Subsequently, the lagoons inland of these were gradually transformed, by sediment infilling, into swamps at the beginning of Roman Age, and even into solid ground at present day.

3 The four main sites in the coastal plain are the protohistorical site Montarice, the Roman colonial centre Potentia, and the medieval towns Potenza Picena and Porto Recanati (fig. 2).

4 Complementary methods of investigation of both archaeology and the geosciences need to be combined to achieve a better assessment of the evolution of the coastal plain.

5 Archaeological surface survey implies field walking with interdistance of 10 to $15 \mathrm{~m}$ in search of artefacts, and careful registration of the results with aid of handheld GPS, index cards, and land registry maps into a database using Windows Access and into a GIS using ArcView 3.2 software. All artefacts are washed, numbered, and sorted; some are depicted. Forty-seven sites have been found, which can be subdivided into five groups (fig. 2).

6 Prospection through interpretation of oblique low-altitude aerial photographs implies the search for soil marks and crop marks, leading to the discovery of new sites, as well as a better understanding of the known sites, especially the Potentia city plan and the Montarice outlay. Furthermore, the aerial photographs aid in detecting palaeo-channels (fig. 4). As many as 2,300 low-altitude aerial photographs have been taken in different seasons in order to better assess the impact of hydrological, meteorological and phenological conditions on the visibility of crop marks and soil marks.

7 Prospection of the subsurface by means of hand augering (fig. 6 and 7), interpretation of conventional vertical aerial photographs and geomorphological study aim at checking the position of palaeo-channels of the Potenza river (fig. 6, P1 \& P2) and beach ridges, and their associated sedimentary environments. ${ }^{14} \mathrm{C}$ datings and mollusc analysis enable us to determine the age and nature of the palaeo-environment. Based on the fluvial type profile near a Roman bridge (fig. 6 and 7, augering 1) and aerial photographs, the course of two palaeo-channels has been reconstructed, the one beneath the Roman bridge being active until $630 \pm 25 \mathrm{BP}$ or $1290-1400 \mathrm{Cal} \mathrm{AD}\left({ }^{14} \mathrm{C}\right.$ dating on charcoal in the gravel lag; 2 $\sigma$-interval, KIA-19509). Two beach ridges and the clay cover in between are located as well by the hand augerings.

8 Prospection of the subsurface by means of geoelectrical profiling and sounding (fig. 9 and 10) is used in two ways: firstly to find the best augering locations and thus reduce time and cost; secondly to reconstruct more accurately the lateral continuity of sedimentation units, as profiling allows to survey transects instead of points. A simple pole-pole array using a terrameter proved sufficient (fig. 8).

9 Each of these prospecting methods has its advantages and disadvantages. Archaeological survey allows fast, low-cost and non-destructive assessment of the human occupation, but lacks possibilities for investigation of relationships with the surrounding landscape. Both low-altitude and high-altitude aerial photograph interpretation can fill this methodological gap, although a ground based check of soil marks, crop marks, and related features is still required, which is in turn 
provided by hand augering and geoelectrical prospection. To avoid circular reasoning, the archaeological and geomorphological teams work strictly independent on site, but they regularly exchange and confront the obtained results.

This research resulted in an evolution scheme of the Potenza coastal plain starting from Roman Age, when the port town Potentia was situated on a beach ridge that formed during or before the Iron Age (900-268 BC). M. Coltorti (1997) mentions a change of river regime from incising to aggrading in the late Roman Age. The resulting sediment surplus is at first deposited in the lagoons and marshes inland of the beach ridges, and subsequently contributes to the progradation of the coastline, which reaches its actual position in the $11^{\text {th }}$ century. In the $14^{\text {th }}$ century, the Potenza river probably (research in progress) shifted southward by avulsion, to a course that is clearly visible on aerial photographs. Finally, man has diverted the Potenza river to its actual artificial course in the north of the coastal plain, incising the alluvial fan of a tributary.

Figure 1. Localisation de la zone étudiée en Italie et dans les Marches.

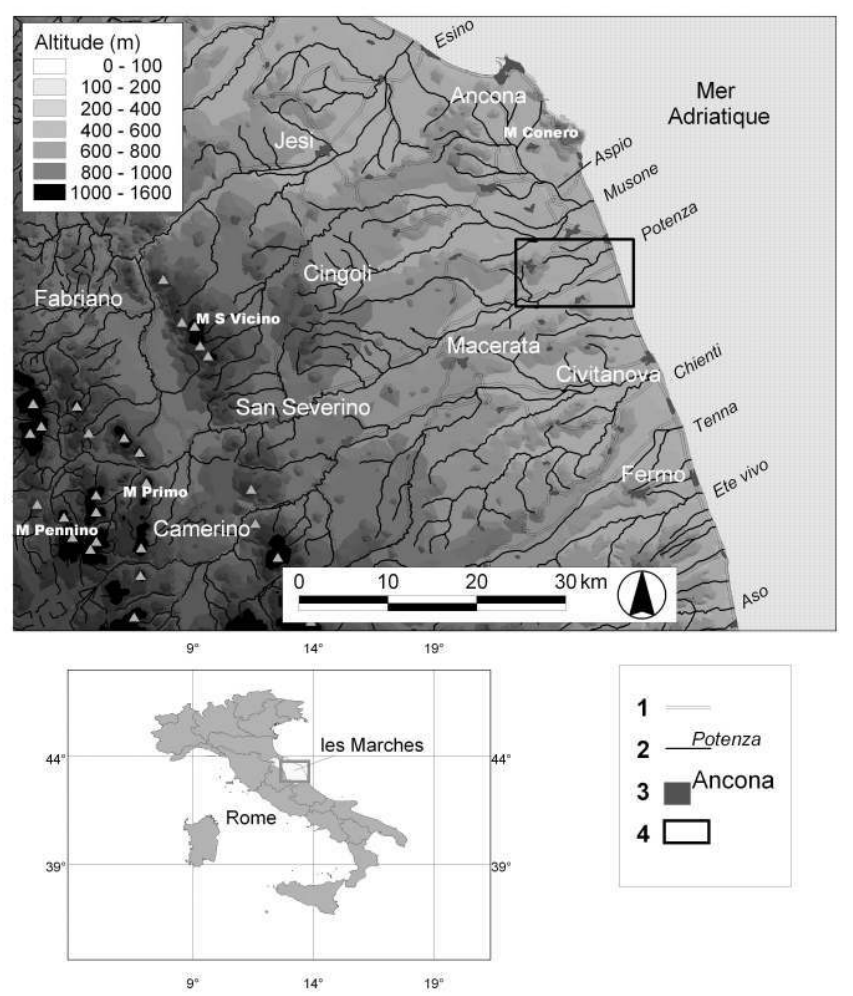

1 : réseau routier ; 2 : fleuves ; 3 : agglomérations ; 4 : zone d'études plaine côtière DONNÉES DE BASE FOURNIES PAR L'UFfICIO CARTOgRAfIA E INFORMAZIONI TERRITORIALI, REgIONE MARCHE

\section{Introduction}

La plaine côtière du fleuve Potenza a été étudiée dans le cadre du projet géoarchéologique «The Potenza Valley Survey. From Acculturation to Social Complexity in Antiquity: A Regional Geo-Archaeological and Historical Approach » depuis l'année 2000. Ce projet se concentre sur deux objectifs : la reconstitution de l'occupation humaine et de l'évolution du paysage durant le premier millénaire qui a précédé notre ère et le millénaire qui l'a suivi; et la mise en place d'une méthodologie géo-archéologique. 
L'orientation multidisciplinaire du projet se traduit par la collaboration entre le Département d'archéologie et histoire ancienne de l'Europe et celui de géographie de l'université de Gand (Belgique).

Les dernières années, l'accent des recherches archéologiques méditerranéennes s'est déplacé d'excavation à la prospection, et de strictement archéologique à géoarchéologique. Des projets interdisciplinaires, découlant de cet esprit, complémentent les recherches classiques. Ainsi le projet Potenza Valley Survey s'est établi en collaboration avec le bureau archéologique régional (Soprintendenza per i Beni Archeologici delle Marche) : le bureau archéologique fournit les rapports de fouilles des grands sites d'occupation humaine de la région et l'état des connaissances archéologiques, le Potenza Valley Survey ajoute les résultats de l'implémentation des nouveaux techniques et tente à répondre aux questions non partagées, concernant la situation des sites par rapport à l'hydrographie, la présence de sites archéologiques plus petits, les divers aspects du paysage dans le passé, l'influence de la géomorphologie sur les réseaux routiers, etc.

Dans le secteur de l'embouchure se trouvent quatre grands sites d'occupation humaine : le site protohistorique de Montarice, le centre colonial romain de Potentia, et les villes médiévales de Potenza Picena et Porto Recanati (fig. 2). Montarice, bien que fondé pendant l'âge du bronze, fut important avant tout à l'âge du fer comme site d'altitude préurbain de l'élite des Piceni, du fait de sa position à l'extrémité est de l'interfluve entre les fleuves Potenza et Musone. Potentia a été fondée en l'an 184 avant notre ère. La ville a connu un essor entre le $\mathrm{III}^{\mathrm{e}}$ et le $\mathrm{VI}^{\mathrm{e}}$ siècle de notre ère, malgré une crise pendant le $\mathrm{V}^{\mathrm{e}}$ siècle (peut-être à cause de l'instabilité du littoral et la formation de marais). Un déclin au $\mathrm{VI}^{\mathrm{e}}$ siècle aboutit à l'abandon total du site au $\mathrm{VII}^{\mathrm{e}}$ siècle. Ville portuaire au moment de sa fondation, Potentia est aujourd'hui située à $300 \mathrm{~m}$ de la mer. Potenza Picena et Porto Recanati furent construites respectivement au $\mathrm{x}^{\mathrm{e}}$ et $\mathrm{XIII}^{\mathrm{e}}$ siècle, l'un sur une butte de l'interfluve sud du fleuve Potenza, l'autre au pied du versant de vallée au nord. Néanmoins, une église romane précéda la ville de Porto Recanati au XI ${ }^{e}$ siècle. 
Figure 2. Localisation des sites archéologiques repérés pendant le projet Potenza Valley Survey.

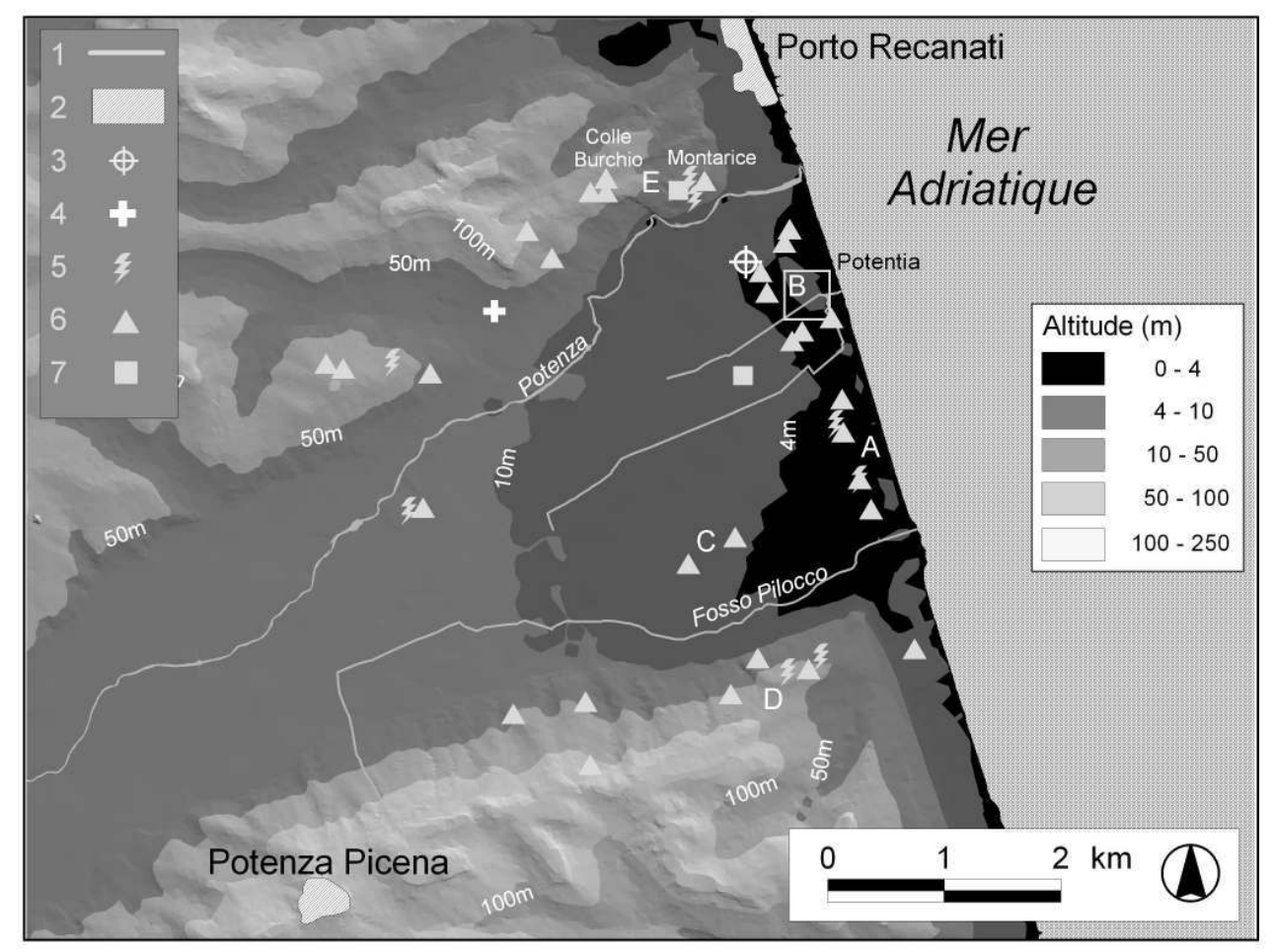

1 : cours d'eau ; 2 : agglomération ; 3 : sondage G. Cilla \& F. Dramis (1999); 4 : sites âge de pierre ; 5 : sites protohistoriques ; 6 : sites romains ; 7 : sites médiévaux ; $A, B, C, D, E$ : groupes de sites

\section{Cadre géomorphologique}

Le fleuve Potenza ( $88 \mathrm{~km}$ de long) est un de ces cours d'eau parallèles qui dévalent le versant oriental de l'Apennin d'Ombrie et des Marches (fig. 1). Son bassin versant élongé $\left(775 \mathrm{~km}^{2}\right)$, tout entier dans la province de Macerata, est perpendiculaire aux principales unités de relief depuis la ligne de faîte de l'Apennin jusqu'au rivage de la mer Adriatique. Il recoupe : les deux chaînons, allongés du nord-nord-ouest au sud-sud-est, inscrits dans les calcaires et les marnes mésozoïques plissés, le piedmont caillouteux constitué de cônes de déjection (étalés au pied de la montagne), l'ensemble de collines taillées dans les dépôts détritiques plio-quaternaires (argiles, sables et conglomérats) de l'avant-pays adriatique (Servizio geologico d'Italia, 1967). Ceux-ci de plus en plus récents d'ouest en est présentent une disposition monoclinale témoignant d'une mise en place par progradation et basculement au fur et à mesure du soulèvement quaternaire (commencé il y a environ 0,6 millions d'années, Ambrosetti et al., 1982).

De part et d'autre de la plaine côtière du fleuve Potenza, large de quatre kilomètres, les collines culminent à 150-200 m d'altitude environ. A proximité du littoral, elles sont bordées par une terrasse marine, large de $500 \mathrm{~m}$ environ, située 5-6 $\mathrm{m}$ au-dessus du niveau de la mer et limitée à son bord interne par une falaise morte. Cette terrasse marine est attribuée par M. Coltorti (communication personnelle) au «sintema del fiume Musone (MUS) ", considéré d'âge Holocène. Il est probable que l'origine soit à corréler avec la surrection tectonique quaternaire de la côte. Une barre sableuse borde la terrasse marine et la plaine côtière du fleuve Potenza. L'apport de sédiments côtiers dans les 
Marches à présent ne provient plus des fleuves, mais émane essentiellement du massif du Mont Conero, au sud d'Ancône. Le principal vent redistribuant en Adriatique est le sirocco, qui provient du sud-est. Ainsi la côte basse au tracé rectiligne au sud du massif du Mont Conero (fig. 1) est en érosion, contrairement aux côtes au nord d'Ancône (Curzi, 1986). Des inondations, par exemple en décembre 1982 et en novembre 1999, ont intéressées une large partie de la plaine côtière jusqu'à présent.

Les géologues et géomorphologues se sont appuyés sur la stratigraphie du sous-sol pour leurs contributions à la reconstruction de l'évolution holocène des plaines côtières de la région des Marches. Dans la plaine côtière du fleuve Potenza, un sondage à $50 \mathrm{~m}$ de profondeur effectué par G. Cilla et F. Dramis (1999; fig. 2 \& 3), à environ $500 \mathrm{~m}$ de son embouchure, a montré que la phase érosive est finie bien avant $9430 \pm 90$ ans BP (il n'est pas clair s'ils parlent d'une date calibrée ou pas), dans le tardiglaciaire, comme l'attestent les dépôts limono-sableux et tourbeux caractéristiques d'un fleuve méandriforme à cette époque. Á la fin de cette phase érosive et à l'emplacement du littoral actuel, le paléoPotenza - alimentant le fleuve Po qui débouchait au milieu de la Mer Adriatique - se trouvait alors à 46 m sous le niveau actuel de la mer (fig 3 ; Cilla \& Dramis, 1999).

Figure 3. Sondage effectué par Cilla \& Dramis (1999).

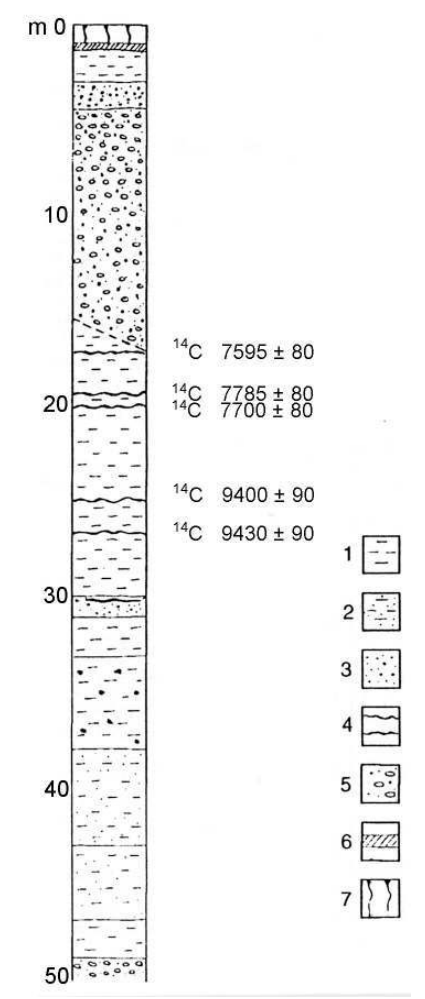

1 : argile limoneuse $; 2$ : limon argilo-sableux ; 3 : sable ; 4 : tourbe $; 5$ : gravier et sable ; 6 : niveau anthropique romain ; 7 : sol actuel

$17 \mathrm{Au}$ début de l'Holocène, la transgression flandrienne forçait le littoral à former une côte du type atlantique (ria) pénétrant sur quelques kilomètres à l'intérieur du pays (Coltorti et al., 1991), avec une alternance de caps et de petites baies près des embouchures des fleuves (Coltorti, 1997). Le bon état de conservation des sédiments adriatiques indique une submersion rapide par une élévation croissante du niveau de la mer entre 11000 et 6000 ans Cal BP (Coltorti, 1997). Le maximum de la transgression marine est daté à 5000 
ans Cal BP par des sédiments lagunaires dans les sondages (Corregiari et al., 1996). La transgression est également attestée dans la région des Marches par les fragments d'orme lisse (Ulmus laevis) repérés dans la plaine côtière du fleuve Foglia, dans des dépôts fins déposés sur la surface d'érosion du Pléistocène supérieur (Gori, 1988). Une sédimentation plus grossière a continué durant la période suivante jusqu'à environ $3970 \pm 60$ ans BP (Cilla \& Dramis, 1999; il n'est pas clair s'ils parlent d'une date calibrée ou pas). Une sédimentation fine plus lente lui a succédé. À ce moment, la dérive littorale provoque le développement de flèches littorales en direction NNO depuis la terrasse marine au pied de la falaise au sud de la plaine côtière. L'ensemble des flèches littorales partant de la terrasse marine engendre des cordons littoraux. Le trait de côte est rectiligne dans le III siècle (Ortolani \& Alfieri, 1978). Au côté terre des cordons se forment par conséquent des lagunes qui évoluent lentement en marais, à l'origine de la malaria durant la période romaine (Ortolani \& Alfieri, 1947). Elles se remplissent ensuite de sédiments alluviaux au Bas Moyen Âge (cf. Baldetti et al., 1983).

La situation du fleuve Potenza au pied du versant nord de la vallée est remarquable par comparaison avec tous les autres fleuves de la région des Marches, sauf le fleuve Musone, qui sont plaqués précisément contre le versant sud de leur vallée. Les sources écrites témoignent d'une déviation artificielle $d u$ fleuve Musone pour des raisons d'assainissement (Ortolani \& Alfieri, 1947; Cencini \& Varani, 1991; Nanni \& Vivalda, 1987). Le fleuve Potenza est cité par M. Buli \& M. Ortolani (1947), qui ont découvert une lettre du Pape Grégoire IX (1170-1241) adressée aux habitants de Porto Recanati et ordonnant d'aménager une confluence entre les fleuves Potenza, Musone et Aspio (immédiatement au nord du fleuve Potenza ; fig. 1), aussi que la construction d'un port à l'embouchure. Bien que le projet ait été abandonné en 1474 après de longues intervalles d'inactivité, ce document confirme qu'il avait été envisagé. N. Alfieri (Ortolani \& Alfieri, 1947) a reconnu la morphologie de deux deltas morts dans la plaine côtière, ce qui, en combinaison avec la situation à présent, laisse supposer des bras morts du fleuve.

\section{Méthodes mises en oeuvre en géoarchéologie}

19 Les plaines côtières du pourtour méditerranéen sont influencées dans leur évolution géomorphologique par de l'action humaine considérable depuis la Protohistoire (Leveau et al., 1999 ; Vermeulen \& De Dapper, 2000).

L'étude de l'évolution holocène d'une plaine côtière méditerranéenne, dont la topographie est peu contrastée, requiert une prospection de surface selon une méthodologie géo-archéologique qui combine des méthodes complémentaires de prospection. La vision d'ensemble, initialement fondée sur l'examen des documents cartographiques et une importante documentation écrite, a été progressivement testée et affinée par les investigations de terrain, menant parfois à une réévaluation considérable des hypothèses.

21 Une prospection archéologique de surface et une campagne de forages à la tarière ont été ensuite entreprises simultanément. La première a servi à mieux cerner la répartition spatiale des sites d'occupation. La seconde a permis de percer la stratigraphie et donc l'évolution des milieux sédimentaires. La prospection archéologique de surface reste cependant insuffisante pour restituer l'extension spatiale des sites plus étendus comme Potentia et Montarice et pour détecter les structures ensevelies. Cette lacune peut être 
comblée par une vue aérienne. Les photographies aériennes verticales permettent d'obtenir une vue générale tandis que les photographies aériennes obliques prises à basse altitude permettent d'examiner des détails près des sites.

L'apport de l'étude géomorphologique (analyse de la microtopographie et prospection des sédiments affleurants) a été suivi de forages à la tarière, dont la localisation a été déterminée grâce à l'analyse des photographies aériennes et des cartes topographiques. Le principal inconvénient des forages, qui se limitent aux seules observations ponctuelles, peut être contourné par la réalisation de transects qui permettent de cerner des transitions, et notamment par la prospection électrique de surface. Afin d'éviter des raisonnements circulaires, les équipes géomorphologiques et archéologiques ont travaillé de façon indépendante. Des séances régulières d'échange et de confrontation des résultats permettent cependant d'ajuster les hypothèses de travail et d'orienter la recherche géo-archéologique.

\section{La prospection archéologique de surface}

La prospection archéologique de surface a été effectuée dans une bande de $3,9 \mathrm{~km}^{2}$ dans la plaine côtière. Elle sert à compléter les connaissances de la Soprintendenza per $i$ Beni Archeologici delle Marche, qui se limitent aux grands sites, avec les petits sites. La prospection a été réalisée entre la fin de l'été et le début de l'automne sur des champs labourés. Elle implique que ces terrains soient soigneusement prospectés par chercheurs séparés de 10 à $15 \mathrm{~m}$, à la recherche d'artéfacts, tessons, éclats et autres objets liés à l'occupation humaine. Les concentrations d'artéfacts ou les artéfacts spécifiques comme les armatures sont localisées grâce à un GPS de poche Garmin GPS 12 et ensuite examinées plus en détail. Tous les transects et les concentrations fouillés sont identifiés sur une fiche, référencés sur le cadastre et finalement incorporés dans une base de données Windows Access afin de pouvoir les exploiter dans le SIG ArcView GIS 3.2. Chacun des objets trouvés est lavé, numéroté et classé et les plus intéressants sont dessinés.

La classification commence par une distinction fondée sur la nature du matériau : roche, métal, verre, céramique. La céramique est subdivisée en terre cuite architecturale, poterie diagnostique et non diagnostique. Compte-tenu des traces d'usure et de la fragmentation des artéfacts, la classification chronologique du matériel céramique, presque uniquement fondée sur la composition de l'argile, se fait par comparaison avec une collection de référence déjà élaborée par les chercheurs italiens. Il est évidemment impossible de proposer une chronologie très précise à partir de ces prospections de surface. Tous les objets repérés sont mis en dépôt à la Soprintendenza per i Beni Archeologici delle Marche, l'organisme de coordination des recherches archéologiques dans la région des Marches.

Les sites repérés par l'équipe archéologique peuvent être classés en cinq groupes selon leur position géographique (fig. 2) : un groupe de sites alignés à $300 \mathrm{~m}$ de la côte actuelle, au sud de Potentia; un autre autour de Potentia; un troisième constitué de deux sites romains au-dessus de l'isohypse de $5 \mathrm{~m}$ (aucun site n'ayant été repéré dans le reste de la plaine alluviale); un quatrième groupe comprenant des sites protohistoriques et romains épars et une villa romaine sur le versant sud; enfin un cinquième ensemble de sites protohistoriques et romains sur l'interfluve nord, autour de Montarice. 


\section{La prospection par photos aériennes}

Deux types de photos aériennes sont utilisés : photos aériennes obliques de basse altitude et photos aériennes verticales de haute altitude. Les photographies aériennes obliques de basse altitude aux grandes échelles sont complémentaires des photographies verticales de haute altitude prises par l'Institut Géographique Militaire Italien à l'échelle de 1/30 000 . De point de vue géomorphologique, les prises de vue verticales permettent la détection des paléochenaux du fleuve Potenza et des anciens tracés de la côte (voir infra), tandis que les vues obliques permettent de discerner l'extension des dépôts fluviatiles et des morphologies côtières (les cordons littoraux) dans la zone autour de la ville de Potentia, et d'étudier ces traits en rapport avec l'expansion des structures urbaines.

L'acquisition de photographies aériennes obliques de basse altitude pour des fins archéologiques a débuté en 2001 et se poursuit actuellement. Les photographies sont prises d'un petit porteur Cessna très manoeuvrable, à une hauteur de 90 à $150 \mathrm{~m}$, par une caméra Canon $35 \mathrm{~mm}$ SLR munie d'un rouleau de pellicule diapositive Fujichrome 100 ISO. Depuis 2004, une caméra Canon Eos 300D digitale à 6.3 mégapixel effectifs est utilisée. Les campagnes de prises de vue se sont effectuées à différentes saisons, notamment au printemps, en été et au début de l'automne, ce qui permet d'appréhender les effets des conditions hydriques et des différents stades phénologiques de la végétation sur la visibilité des traces archéologiques (Vermeulen et al., 2002). Cette flexibilité de vol fait des photographies aériennes obliques de basse altitude un outil précieux.

La prospection vise à repérer des indices linéaires, ponctuels ou diffus qui traduisent la trace de vestiges archéologiques enfouis par des anomalies du couvert végétal (" anomalies» de végétation; "cropmarks») ou de la couleur des sols (" anomalies» de sols ; «soilmarks »). Les " anomalies» de végétation (fig. 4) sont des dessins issus de la croissance différentielle de plantes cultivées ou non en réponse à des variations spatiales des caractéristiques pédologiques. Des fossés et des puits, souvent comblés par du matériel contenant plus de matière organique, provoquent une croissance plus rapide des végétaux, tandis que les plantes sont au contraire plus petites lorsqu'elles sont localisées au dessus de murs ou carrelages enterrés. Les céréales sont les cultures qui mettent le mieux en évidence ces « anomalies » de végétation. Les « anomalies» de sols (fig. 4) sont principalement provoquées par la mise à l'affleurement de la couche archéologique par les labours, et secondairement par des amas d'artéfacts à la surface du sol. Des fossés et des puits comblés contrastent par leur aspect plus foncé que le sol avoisinant, tandis que des murs apparaissent en traces plus claires (Renfrew \& Bahn, 2000). 


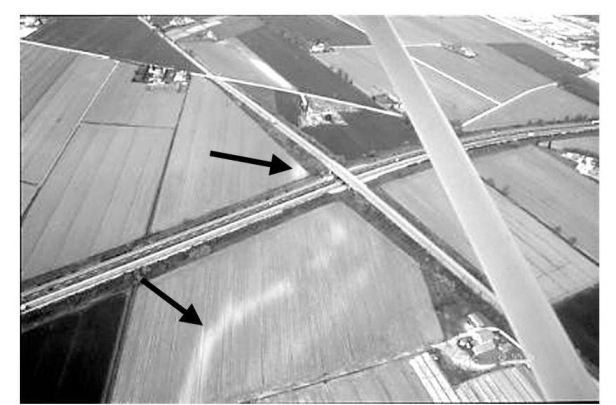

A

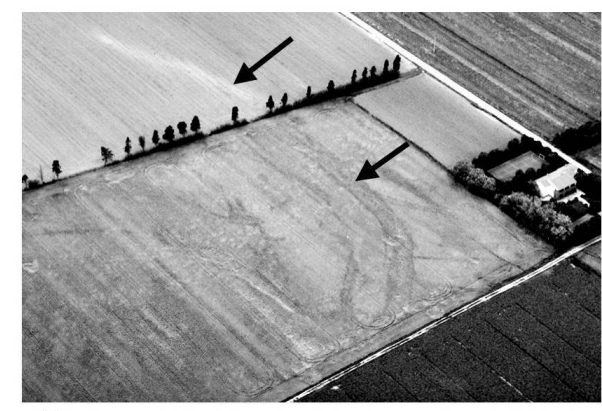

C

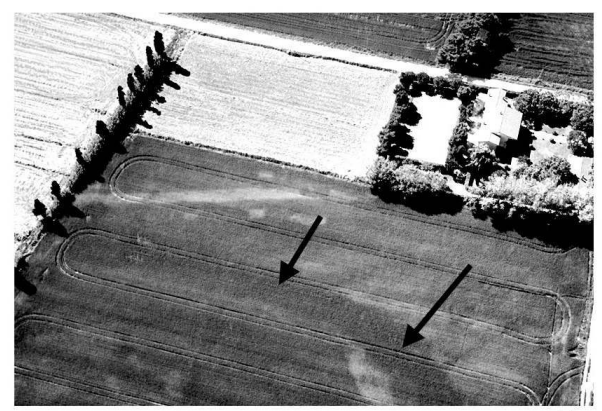

B

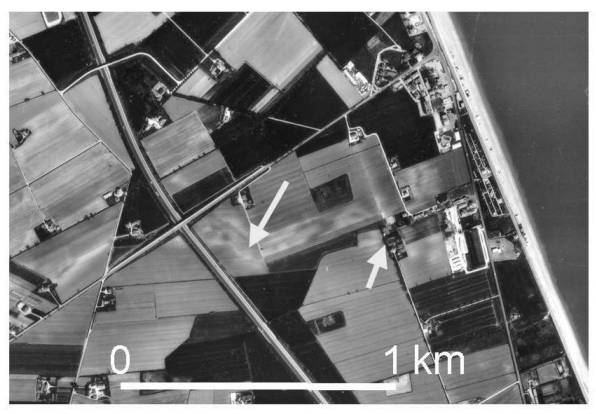

D

Paléochenal 1 révélé par une anomalie des sols (A) et anomalie de végétation (B) et le même paléochenal dans d'autres conditions phénologiques (C); et paléochenal 2 révélé par une anomalie de végétation et de sols sur une vue verticale (D). Les échelles de $A, B$ et $C$ ne peuvent pas être présentées car il s'agit de photos aériennes obliques (avec des grosses déformations) ; le contraste est augmenté pour des raisons de visualisation.

Les 2300 photographies prises jusqu'à présent dans toute la vallée ont permis de découvrir un bon nombre de sites archéologiques inconnus et d'examiner plus en détail les gisements déjà connus. Après vérification sur le terrain, la plupart des " anomalies » de sols se sont avérées coïncider avec des concentrations de débris dans des couches d'occupation humaine, et des zones plus organiques et d'humidité différente. Par l'abondance des débris et leur nature surtout pierreuse et grossière, les traces d'occupation de la période romaine sont les plus visibles. Sur les pentes, la dispersion des débris par le ruissellement et les glissements de terrain ont pu accroître l'extension de ces marques jusqu'à former de larges taches irrégulières. Des marques linéaires indiquent des chemins, des murs, des fossés ou bien des limites de centuriations. Des « anomalies " de végétation ont permis de retrouver l'enceinte du site de Montarice, le plan de ville du site de Potentia, et même quelques monuments funéraires le long des routes menant de Potentia aux villes environnantes.

L'inconvénient principal des photographies aériennes obliques de basse altitude est qu'elles ne peuvent pas être incorporées directement dans un SIG à cause de déformations géométriques. On a pu y remédier par l'utilisation du logiciel Airphoto 2.20 (Verhoeven \& De Vliegher, 2003). Toutes les photos rectifiées géométriquement seront incorporées également dans le SIG ArcView GIS 3.2, afin de produire une carte synoptique à l'échelle de $1 / 10000$. Ainsi un plan détaillé des rues des villes de Potentia a déjà pu être reconstitué (Vermeulen \& Verhoeven, 2004). 
Aujourd'hui le cours inférieur du fleuve Potenza longe le pied du versant nord, mais selon les historiens ceci n'a pas toujours été le cas. N. Alfieri (Ortolani \& Alfieri, 1947) a déjà mentionné les ruines d'un pont romain situées à la localité Casa del Arco, à 1,5 km au sud du cours actuel du fleuve Potenza. La morphologie de deux deltas morts à proximité du pont a permis à cet auteur de supposer un cours romain (fig. 5, A2) et un cours protohistorique (fig. 5, A1). De plus, une inscription sur un pont à Porto Recanati « Arcus hic est pars pontis Potentiae veteris dictae le Fiumarelle » est considérée comme la preuve d'un autre cours dont l'âge estimé remonte à la fin du Moyen Âge (Ortolani \& Alfieri, 1947 ; fig. 5, A3). Malheureusement, la date de l'inscription n'est pas repérée et le tracé se trouve sous les structures urbaines de Porto Recanati. Un autre tracé romain (fig. 5, P1) suggéré par U. Moscatelli \& L. Vettorazzi (1988) et E. Percossi Serenelli (2001) peut être éliminé parce que les auteurs se fondent sur des présumées circulations d'eau souterraine qui en fait ne sont pas liées aux courants de surface. Sur les photographies aériennes, des traces linéaires apparaissent, qui correspondent à des affleurements de graviers, à peine visibles au niveau du sol (fig. 4).

Figure 5. Tracés hypothétiques du fleuve Potenza d'après M. Ortolani et N. Alfieri (1947) et $\mathrm{E}$. Percossi Serenelli (2001) reportées sur un fond topographique actuel.

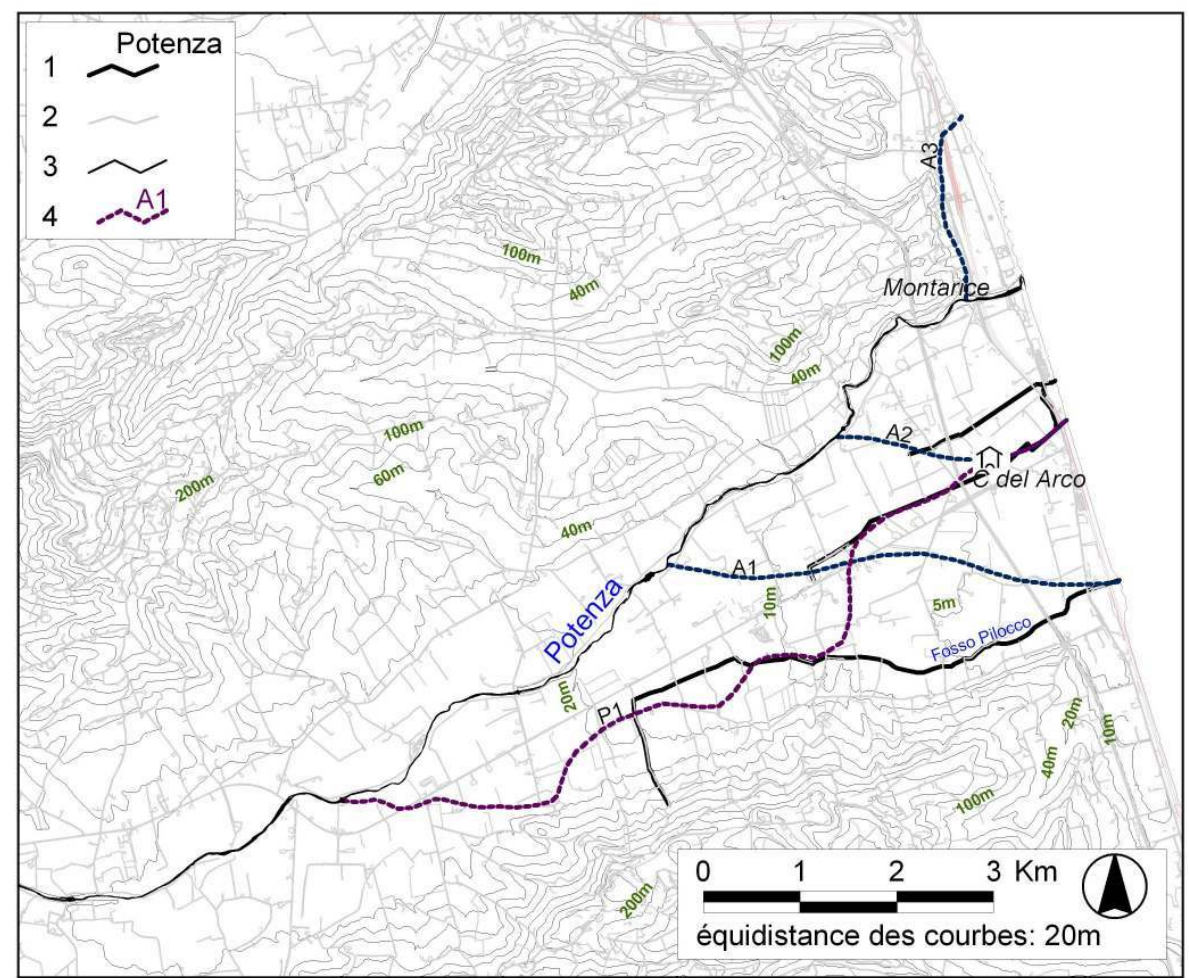

1 : cours d'eau et drains actuels ; 2 : autoroutes et voie ferrée ; 3 : isohypses ; 4 : lits supposés de la période proto-historique (A1), de l'époque romaine (A2 et P1), du Bas moyen Âge (A3).

DONNÉES DE BASE FOURNIES PAR L'UFfICIO CARTOgRAFIA E INFORMAZIONI TERRITORIALI, REgIONE MARCHE. 


\section{La prospection des terrains superficiels par forages à la tarière}

32 Les forages sont réalisés avec une tarière Eijkelkamp, avec des têtes adaptées aux terrains hétérogènes. Ce type de forages implique l'extraction d'un échantillon déstructuré et l'impossibilité de retrouver une stratification laminaire ou autres structures sédimentaires fines. La description des forages inclut : une description de la localisation du forage et les coordonnées ; la profondeur des unités stratigraphiques; la texture ; la couleur selon le code Munsell; les inclusions et autres traits particuliers; la réaction à $\mathrm{HCl}$ et $\mathrm{H}_{2} \mathrm{O}_{2}$; et la profondeur de la nappe phréatique. Un échantillon témoin est prélevé dans chaque unité stratigraphique afin de pouvoir déterminer sa texture précise au laboratoire. En outre des échantillons de matière organique (charbon de bois essentiellement), des mollusques et des fragments de céramique font l'objet d'analyse. L'altitude de chaque point de forage par rapport au réseau géodésique local est mesurée par nivellement. Les levés altimétriques, les localisations et l'ensemble des descriptions permettent d'évaluer la succession et la position des couches sédimentaires (fig. 6 et 7). En ce qui concerne les datations sur charbon de bois, il est bien possible que les échantillons ne soient pas contemporains de la mise en place du sédiment mais bien érodés d'un dépôt plus vieux. Cependant, il est supposé que le laps de temps ne soit pas assez grand, vu que les datations donnent des résultats assez récents (qu'on ne prévoit pas dans le cas de charbons vieux retransportés) et vu que les datations sont cohérentes (succession de dates dans un profil, contemporanéité d'échantillons dans une identique position). 
Figure 6. Localisation des forages à la tarière et sondages géoélectriques dans la plaine côtière du fleuve Potenza.

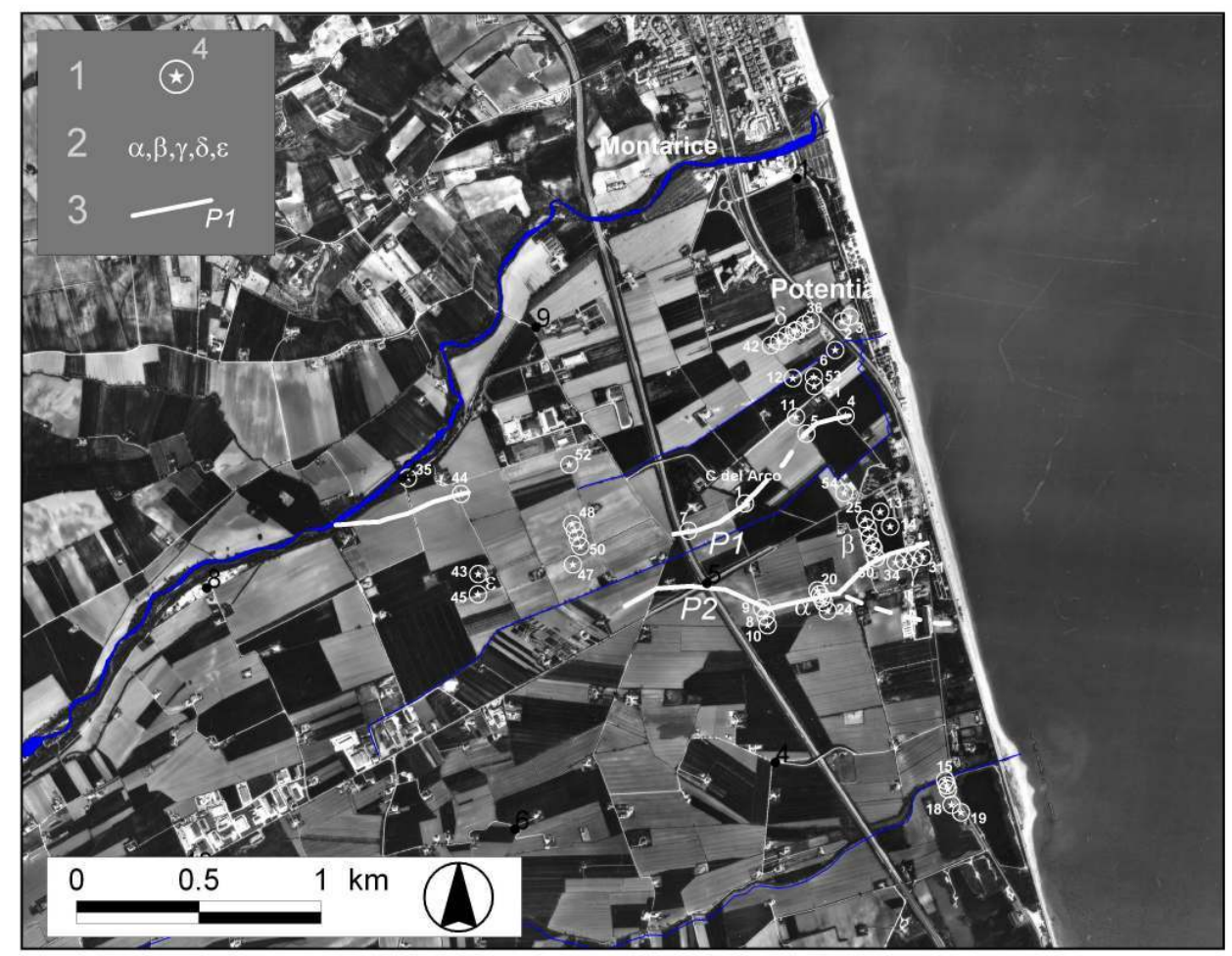

1 : forages à la tarière ; 2 : transects géoélectriques ; 3 : paléochenal 1 (P1) et paléochenal 2 ( $P 2)$. Les forages à la tarière 1, 4, 5, 7 et 44 correspondent au paléochenal 1 ; les forages à la tarière 8, 9, 10, 20 à 24 et 44 ( ?) correspondent au paléochenal 2 ; les forages à la tarière 2, 6 et 36 à 42 sont situés sur le cordon littoral ; les forages à la tarière 11 à 19, 25 à 30, 31à 34, 43 et 45 se trouvent dans la lagune (sédiments d'inondation) ; le forage à la tarière 3 correspond à la dépression entre les cordons littoraux ; et le forage à la tarière 35 présente des sédiments d'inondation du chenal actuel. Arrièreplan : photographie aérienne rectifiée de l'IGM n 1150. vol du 30 mars 1994. 
Figure 7. Résumé des forages à profils type.

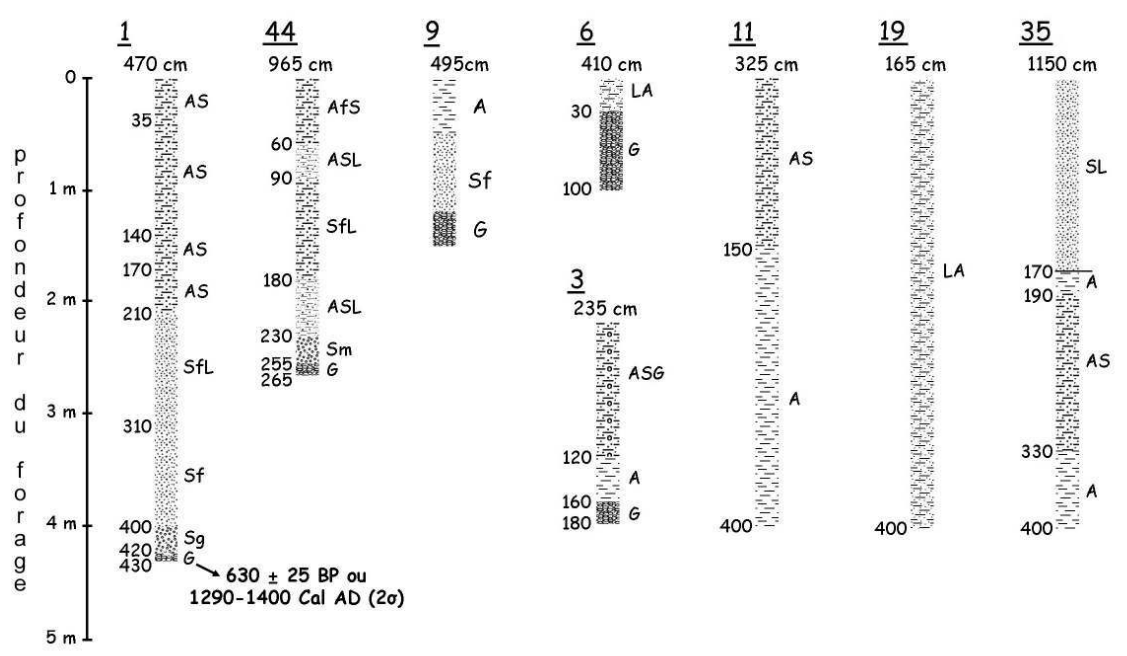

Les chiffres en cm représentent la hauteur au-dessus du niveau de la mer de l'apex du forage. A : argile; AfS : argile fin sableuse ; AL : argile limoneuse; ALS : argile limo-sableuse ; AS : argile sableuse ; ASG : argile sablo-graveleuse ; ASL : argile sablo-limoneuse ; G : gravier ; GS : sable graveleux ; L : limon ; LA : limon argileux ; LfS : limon fin sableux; LS : limon sableux ; LSA : limon sablo-argileux; S : sable ; Sf : sable fin ; SfL : sable fin limoneux; SG : gravier sableux; Sg : sable grossier ; SgA : sable grossier argileux ; SL : sable limoneux ; Sm : sable moyen.

33 Un premier forage a été effectué au site Casa del Arco (fig. 6 et 7, forage 1), où les ruines d'un pont romain de la route Pausulae-Potentia marquaient la présence supposée du lit romain du fleuve Potenza. Le profil sédimentaire montre en effet un grano-classement normal : graviers et sables grossiers à la base (dès $400 \mathrm{~cm}$ de profondeur), passant à des sables fins et des limons (de 400 à $210 \mathrm{~cm}$ ) et finissant par des argiles sableuses (les $210 \mathrm{~cm}$ supérieurs). Ce grano-classement normal indique des sédiments transportés et déposés dans un chenal par de l'eau courante à vitesse faiblissante et un colmatage final par une nappe argileuse alluviale. Contrairement aux hypothèses antérieures selon lesquelles le fleuve Potenza coulait ici uniquement à l'époque romaine, les datations au ${ }^{14} \mathrm{C}$ sur quelques fragments de charbon de bois dans les graviers à la base de ce profil type ont montré que ce lit était encore actif jusqu'à $630 \pm 25$ ans BP (KIA-19509), soit à 1290-1400 Cal AD (2б). La même succession de sédiments a été retrouvée aux points de forage numéros 4, 5 et 7 (fig. 6). Ces derniers se trouvent dans le même paléochenal 1 (fig. 6, P1; fig. 4 , $\mathrm{A}$ et $\mathrm{B}$ ) détecté à partir des clichés aériens. Ces observations confirment donc l'existence du paléochenal supposé.

Le paléochenal 2 (fig. 6, P2 ; fig. 4, C et D) est confirmé de la même façon (interprétation de photos aériennes, forages, profilages géo-électriques) par les forages numéros 8 à 10 (fig. 6 et 7) et 20 à 24 (profil géoélectrique $\alpha$ ). Le cordon littoral sur lequel la ville de Potentia est située a été attesté par les forages numéros 2, 6 (fig. 6 et 7) et 36 à 42 (profil géo-électrique $\delta$ ), tandis que le forage numéro 3 (fig. 6 et 7) montre les sédiments de la dépression entre ce cordon littoral et son homologue actuel. Tous les autres forages, sauf les numéros 35 et 44 (mentionnés ci-dessous), montrent des argiles et limons homogènes qui pourraient indiquer, non seulement des sédiments lagunaires ou marécageux, mais encore des sédiments d'inondation fluviatile. 

d'émission $\mathrm{A}$ et une de mesure de potentiel $\mathrm{M}$, sont posées sur le tracé de mesure à une distance $\mathrm{L}$ qui détermine la profondeur de pénétration, estimée à $\mathrm{L} / 2$; on mesure la résistivité apparente au milieu de ces deux électrodes. Deux électrodes extérieures, une électrode d'émission $\mathrm{B}$ et une électrode de mesure de potentiel N, sont posées à l'infini (> 20 L). Cette méthode a été appliquée avec succès à la recherche de vestiges archéologiques (De Breuck \& Beeuwsaert, 1997). 
Figure 8. Schéma de la configuration pôle-pôle.

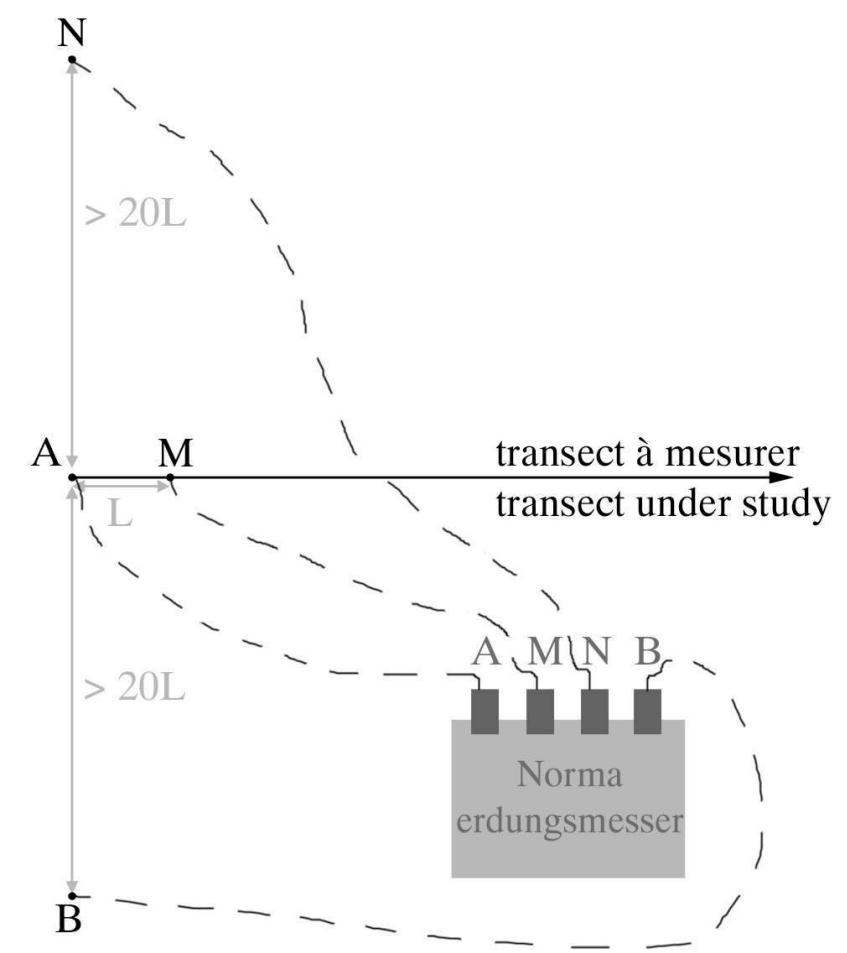

A \& B : électrodes d'émission ; $M$ \& N : électrodes de mesure de potentiel (cf. configuration Wenner). A \& $M$ : électrodes intérieures ; $B$ \& $N$ : électrodes extérieures.

Avec cette configuration la résistivité apparente devient :

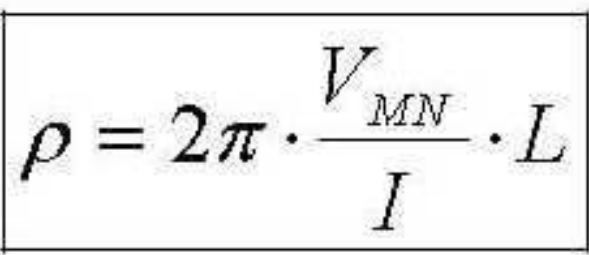

avec : $r$ = résistivité apparente au milieu entre $M$ et $N(W \cdot m), V_{M N}=$ potentiel entre $M$ et $N(V), I=$ intensité du courant $(A)$ et $L=$ distance entre $M$ et $N(m)$.

La technique pôle-pôle adaptée a quelques avantages vis-à-vis des méthodes classiques comme le dispositif Wenner : on peut utiliser un résistivimètre compact et léger, ici un Norma Erdungsmesser D3950 qui mesure directement la valeur $\mathrm{V}_{\mathrm{MN}}$ /I, et des tiges métalliques comme électrodes, car un courant alternatif suffit pour des sondages peu profonds. En outre le dispositif pôle-pôle nécessite des distances trois fois plus petites que le dispositif Wenner pour contrôler une profondeur analogue (Brass et al., 1981) et il ne faut déplacer que deux ou bien une seule électrode par mesure. Sur des champs larges et libres d'obstacles la procédure nous a permis de mesurer un transect de $150 \mathrm{~m}$ de longueur avec une distance entre les électrodes de $2 \mathrm{~m}$ en une demi-journée, avec trois personnes.

Néanmoins on ne peut observer que des phénomènes peu profonds, jusqu'à environ $5 \mathrm{~m}$, à cause de l'emploi de courant alternatif qui souffre de l'effet pelliculaire et de l'induction dans les câbles utilisés. La technique ne donne pas des résultats absolus, car chaque fois 
qu'on déplace les électrodes extérieures, l'échelle des résistances apparentes change, probablement à cause d'une forte impédance.

42 L'expression graphique des résultats est adaptée à ce dernier inconvénient. On pourrait représenter les résultats sous forme de $z$-scores ( = les résultats, normalisés avec la moyenne et l'écart type du transect entier), mais dans ce cas il est impossible de comparer les transects examinés. La meilleure méthode consiste à représenter $\Delta \rho$, étant la différence entre la résistivité apparente mesurée et la résistivité apparente minimale du transect.

Le choix des transects est fondé sur les investigations précédentes et sur l'analyse des photographies aériennes. La distance L la plus appropriée pour mesurer la variation lithologique des couches superficielles est déterminée empiriquement (ici $2 \mathrm{~m}$, donc une profondeur de pénétration de l'onde de l'ordre de $1 \mathrm{~m}$ ), en testant les résistivités apparentes de quelques points déjà examinés par des forages à la tarière. Partant des observations de terrain et au vu des résultats, les points aux résistivités les plus représentatives sont signalés. Sur ces emplacements on exécute ensuite des sondages à des distances $\mathrm{L}$ croissantes $(\mathrm{L}=2 \mathrm{~m}, 3 \mathrm{~m}, 4 \mathrm{~m}, 5 \mathrm{~m}, 6 \mathrm{~m})$, pour juger de l'homogénéité du sous-sol. Un forage à la tarière est ensuite effectué. Ainsi les sondages géoélectriques ne servent pas seulement à évaluer les transitions lithologiques entre les forages à la tarière, mais ils permettent aussi de compléter ce réseau d'une manière rapide et efficace.

La prospection a débuté par un transect à travers de la trace linéaire, déjà mentionnée, correspondant à des graviers affleurants mais à peine visibles au ras du sol (fig. 4). Le profil géoélectrique $\alpha$ (fig. 6,9 et 10, forages 20 à 24) montre l'extension latérale des diverses zones sédimentaires, représentées par les plateaux dans le graphique de $\Delta \rho$. On peut corréler ces valeurs de $\Delta \rho$ avec les textures obtenues par les sondages à la tarière. 
Figure 9. Localisation des transects géoélectriques $a, b, g$ et $d$ sur des photographies aériennes obliques.

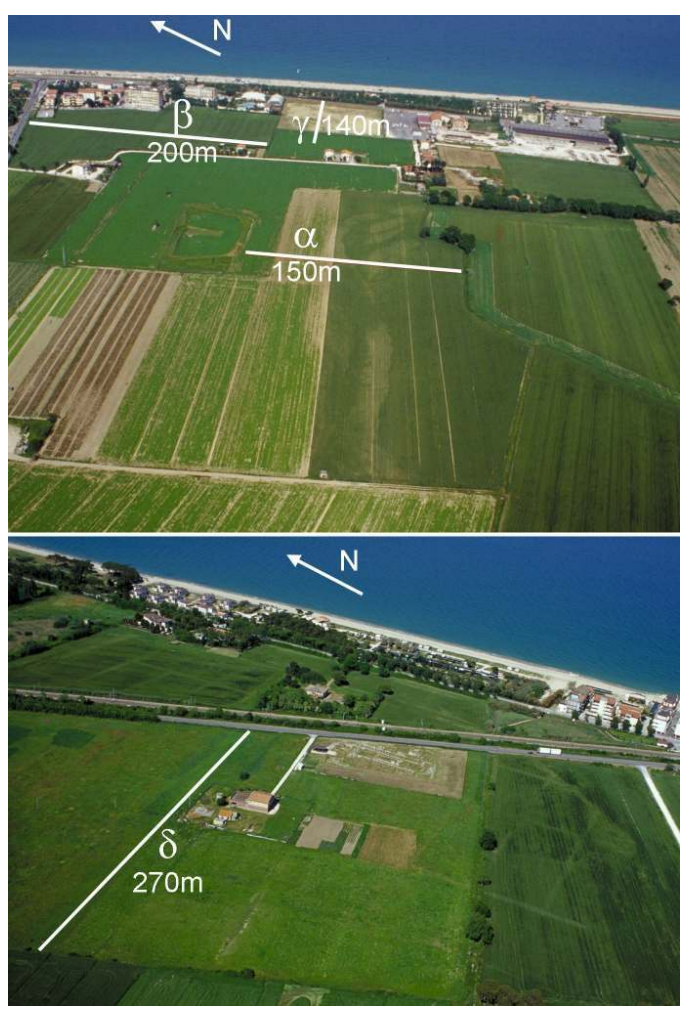

Figure 10. Transects géoélectriques $\mathrm{a}, \mathrm{b}, \mathrm{g}$ et $\mathrm{d}$ et forages correspondants.
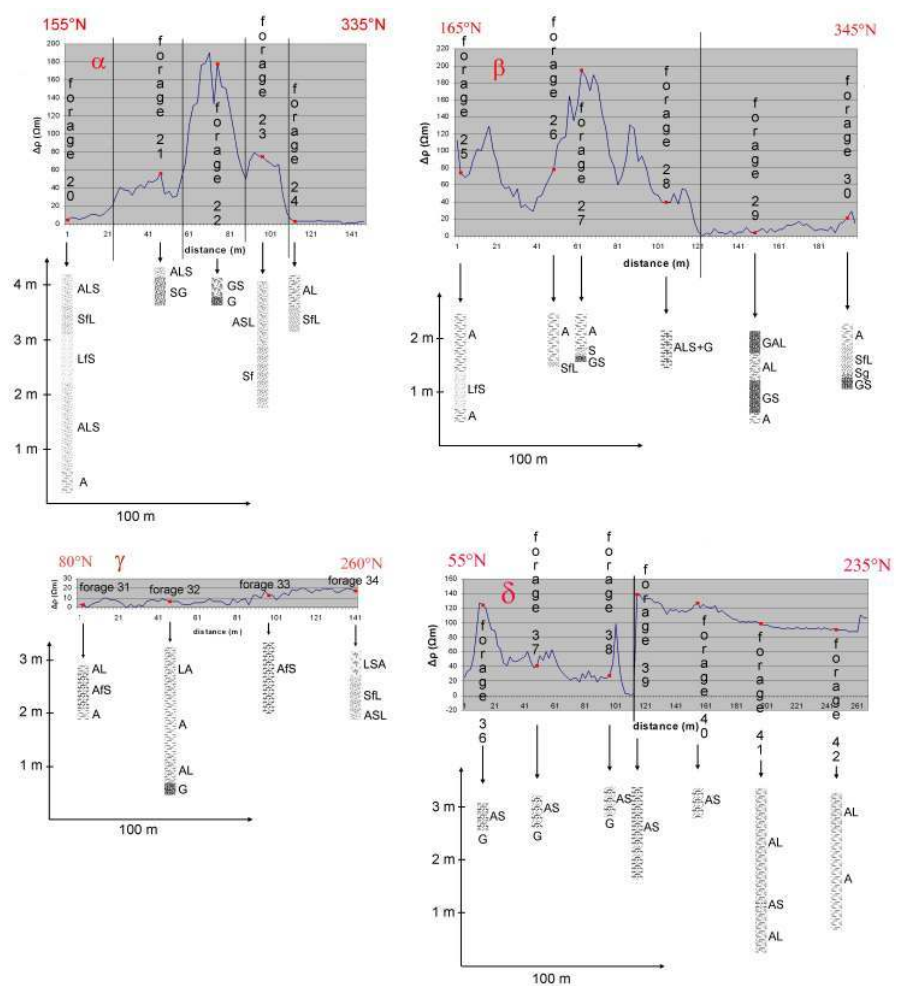

Le transect g montre peu de variation, la stratigraphie se révèle seulement dans les forages. 

le problème de convergence de résistivité de l'argile et du sable imbibé d'eau salée. Le gravier aura une résistivité nettement plus élevée que le sable, qui à son tour aura une résistivité plus élevée que l'argile, ce qui est confirmé par les sondages. Au centre du transect, des graviers $(\mathrm{G})$ sont présents, attestant le centre du paléochenal ; à côté, des sables (S) représentent les bourrelets de berge; les argiles (A) autour signalent les alluvions la plaine d'inondation. Cette morphologie valide l'hypothèse d'un lit abandonné du fleuve Potenza.

Afin de trouver le prolongement de ce lit, un deuxième transect géoélectrique $\beta$ a été examiné au nord-est (fig. 6,9 et 10, forages 25 à 30). Les résultats se sont avérés toutefois moins évidents : on peut supposer l'existence du chenal d'un cours d'eau au niveau de la résistivité maximale correspondant aux graviers et aux sables (forage 27), mais on peut supposer également qu'il s'agit de l'extrémité sud d'un cordon littoral.

Le troisième transect géoélectrique $\gamma$ (fig. 6, 9 et 10, forages 31 à 34), perpendiculaire aux précédents, a confirmé qu'il n'y a plus de cordon littoral au sud du transect $\beta$, car on y trouve essentiellement des limons et des argiles (fig. 10). Ce transect se trouve donc dans la zone d'embouchure du paléochenal mentionné ci-dessus. Ce paléochenal est probablement postérieur à celui attesté dans le forage au niveau du pont romain, car les paléochenaux sont beaucoup plus visibles et les sédiments d'inondation couvrent le chenal du pont. Dans ce cas, on peut estimer l'âge de l'incision de ce paléochenal au XIV ${ }^{\mathrm{e}}$ siècle. Ceci expliquerait aussi que seul le pont romain de Casa del Arco soit conservé, bien qu'une voie menait à Pausulae au sud de Potentia à l'époque romaine.

La position de la côte a également évolué. La ville de Potentia, connue comme port en son temps, est effectivement construite sur une levée linéaire de hauteur et de direction identiques à celles du cordon littoral actuel, c'est-à-dire avec une hauteur au-dessus du niveau de la mer de $2,6 \mathrm{~m}$ à $2,8 \mathrm{~m}$ et une direction de relèvement de $345^{\circ}$ (NNO) à $165^{\circ}$ (SSE). En plus ce cordon est constitué d'une alternation nette de couches (jusqu'à $5 \mathrm{~cm}$ d'épaisseur) et lentilles alternantes de sable grossier et gravier fin, le gravier étant imbriqué. Cette donnée suggère qu'il s'agit d'un ancien cordon littoral. En fait ce dernier proviendrait d'une série de flèches littorales développées à partir d'une falaise par la dérive littorale parallèle à la côte à l'origine de la dérive des sédiments.

Le quatrième transect $\delta$ (fig. 6, 9 et 10, forages 36 à 42) visait à mieux connaître la largeur de ce cordon littoral, mais il a trop souffert de la construction du réseau routier de la ville de Potentia pour être intact. Partant de la présence de Potentia sur la partie nord et le mur d'un four à céramique in situ sur la partie sud, on peut conclure avec certitude que ce cordon littoral délimitait toute la plaine côtière à l'époque romaine. Il était déjà bien en place dans les temps protohistoriques, car un site de cette époque a été repéré sur la partie sud du cordon littoral. En outre, dans la région des Marches, on a repéré des sites protohistoriques d'altitude sur les interfluves en relation avec des sites de poterie sur les cordons littoraux des plaines limitrophes (Luni, 1981 ; Zuffa, 1959). M. Coltorti (1997) mentionne la transformation de régime des fleuves de la région à l'époque romaine, de cours sinueux et incisés à des cours anastomosés et aggradants. Ce phénomène est par ailleurs attesté dans tout le domaine méditerranéen (Bruneton et al, 2001; Marchetti, 2002). En résumé, le sédiment résiduel des fleuves est déposé dans les lagunes et les marais protégés par le cordon littoral. Dès que le remplissage des lagunes et des marais est complété, la côte commence à prograder, jusqu'au littoral actuel sur une distance de 
300 à $500 \mathrm{~m}$. Étant donné que les premières villes côtières de la région, comme Porto Recanati, sont établies au pied des falaises après l'an mil, la progradation est donc à situer entre l'époque de la ville portuaire de Potentia et l'an mil.

Enfin, il fallait déterminer l'endroit exact de la bifurcation entre le cours supposé au XIV ${ }^{\mathrm{e}}$ siècle et le cours actuel. Le cours inférieur du fleuve Potenza actuel semble être artificiel d'après sa position au nord de la plaine côtière, contrairement à celle des autres fleuves de la région. Cette hypothèse est confirmée par des arguments géomorphologiques. À partir d'un point situé à $3,25 \mathrm{~km}$ de l'embouchure actuelle, le lit du fleuve change complètement de morphologie: large et à amples barres graveleuses à l'amont, il se rétrécit en un étroit chenal vers l'aval. De plus, les levés altimétriques ont montré que le fleuve Potenza incise à ce niveau un cône alluvial d'un ruisseau tributaire, dépassant la plaine alluviale environnante d'une hauteur de $2 \mathrm{~m}$. Il restait alors à rechercher le prolongement ouest du lit de l'époque romaine. Le profil géoélectrique $\varepsilon$ (fig. 6, 9 et 10, forages 43 et 45) ne révélait que des sédiments limono-argileux, typiques des inondations fluviatiles. Néanmoins, la localisation du talweg et de la dépression caractérisante a pu être déduite en analysant la microtopographie. Le profil typiquement fluviatile du forage $\mathrm{n}^{\circ} 44$ (fig. 6 et 7) dans le centre de ce talweg supposé confirmait cette hypothèse. Afin de déterminer le moment précis de la déviation, un forage ( $\left.n^{\circ} 35\right)$ a été effectué entre les digues du chenal actuel anthropique. Une césure nette à une profondeur de $170 \mathrm{~cm}$ apparait, avec une couche d'occupation romaine entre 170 et $190 \mathrm{~cm}$ de profondeur. Cette césure indique très probablement la première inondation; la nature sableuse des sédiments semble contredire un processus d'inondation mais il s'agit d'un chenal endigué, ce qui implique des inondations à débits plus énergétiques qui transportent des dépôts plus grossiers. L'âge précis de ces dépôts, pas encore établie, pourrait donner une date ante quem pour la déviation.

\section{L'évolution de la plaine côtière depuis la mise en culture à l'époque romaine}

51 L'évolution de la plaine côtière depuis la mise en culture romaine a débuté avec la ville portuaire de Potentia située au bord de la mer sur un cordon littoral pré-protohistorique. Le fleuve Potenza débouchait $700 \mathrm{~m}$ plus au sud, à $300 \mathrm{~m}$ de la côte actuelle. Peu après, à la fin de l'époque romaine (Coltorti, 1997), le régime des fleuves dans la région change, passant d'une tendance à l'incision à l'aggradation. En un premier temps, les sédiments apportés colmatent les lagunes et les marais protégés par le cordon littoral, puis ils contribuent à la progradation de la côte, qui atteint approximativement sa position actuelle au cours du XI ${ }^{e}$ siècle. Au XIve siècle, le fleuve Potenza se déplace probablement plus au sud par avulsion. Ce cours est encore bien visible sur les photographies aériennes. Néanmoins, cette hypothèse devra être attestée par des datations.

Finalement, l'homme a dévié le fleuve Potenza jusqu'à sa position actuelle au nord de la plaine côtière, en effleurant le pied du versant nord au sommet duquel se trouve le site protohistorique de Montarice, et en incisant le cône alluvial d'un tributaire. L'hypothèse de la position du fleuve Potenza remontant à la protohistoire est rejetée par cet argument. La plaine côtière a été soumise à des inondations périodiques, situation qui continue jusqu'à l'époque récente, les derniers évènements datant de 1982 et de 1999. Par 
conséquent l'expansion urbaine de la ville romaine de Potentia était contrôlée et limitée par les dimensions du cordon littoral sur lequel la ville était fondée.

\section{Conclusion} l'archéologie et des sciences de la Terre, a donné des résultats satisfaisants pour la reconstruction de l'évolution du paysage de la plaine côtière du fleuve Potenza depuis l'époque romaine.

Chaque méthode de prospection montre des avantages et des limites. La prospection archéologique de surface a permis de distinguer d'une façon peu coûteuse, rapide et non destructive, cinq groupes de sites allant du Protohistorique à l'époque romaine. Néanmoins, la signification de ces sites dans le paysage et leurs relations restaient vagues. Les photographies aériennes sont un outil excellent pour détecter des « anomalies » de sols et de végétation qui permettent de comprendre les liens entre l'environnement fluvio-marin et l'organisation des sites et routes. Tandis que les photographies aériennes verticales à haute altitude offrent une vue d'ensemble, les photographies aériennes obliques à basse altitude, par leur grande échelle et leur flexibilité d'acquisition dans le temps et l'espace, donnent des résultats excellents. Il s'agit néanmoins d'interprétations qui, bien que précieuses, doivent être confirmées par des observations de terrain. Les forages à la tarière permettent de détecter la cause des variations pédologiques qui engendrent les «anomalies » de végétation et de sols. Ils permettent aussi de révéler la stratigraphie et donc de comprendre l'histoire des terrains superficiels. Néanmoins les forages ne présentent que des observations ponctuelles. Ce dernier inconvénient peut être remédié avec succès par des sondages et profilages géoélectriques. Cette méthode de prospection ne sert pas seulement à évaluer les transitions lithologiques entre les forages à la tarière, mais elle permet aussi de compléter le réseau de ceux-ci d'une manière rapide et efficace.

Finalement, dans ce genre de projet qui associe des disciplines ressortant d'une culture scientifique différente, sciences naturelles et sciences humaines, il est très important d'éviter des raisonnements circulaires: que le géomorphologue ne voit que ce qui confirme les hypothèse de l'archéologue ou que l'archéologue tire des conclusions seulement fondées sur des arguments géomorphologiques et non confirmés par la réalité des fouilles archéologiques. Ainsi les équipes géomorphologiques et archéologiques de ce projet travaillaient strictement de façon indépendante. Des sessions régulières d'échange et de confrontation des résultats permettaient d'ajuster les hypothèses de travail et d'orienter cette recherche géo-archéologique vers son but final : la reconstruction du jeu d'ensemble Homme-Nature dans l'évolution du paysage de la plaine côtière du fleuve Potenza de 1000 ans avant jusqu'à 1000 ans après notre ère. 


\section{BIBLIOGRAPHIE}

AMBROSETTI P., CARRARO F., DEIANA G. \& DRAMIS F. (1982), Il sollevamento dell'Italia Centrale tra il pleistocene inferiore e il pleistocene medio. Contributi conclusivi per la realizzazione delle Carta Neotettonica d'Italia, Pubbl. N. 513 del Progetto Finalizzato Geodinamica (CNR), pp. 219-223.

BALDETTI E., GRIMALDI F., MORONI M., COMPAGNUCCI M. \& NATALI A. (1983), Le basse valli del Musone e del Potenza nel Medioevo. Archivio Storico Santa Casa, Loreto, 95 p.

BRASS G., FLATHE H. \& SCHULTZ R. (1981), « Resistivity profiling with different electrode arrays over a graphite deposit », Geophysical prospecting, 29, pp. 589-600.

BRUNETON H., ARNAUD-FASSETTA G., PROVANSAL M. \& SISTACH D. (2001), « Geomorphological evidence for fluvial change during the Roman period in the lower Rhone valley (southern France) », Catena, 45, pp. 287-312.

BULI M. \& ORTOLANI M. (1947), « Le spiagge marchigiane », in BEVILACQUA E. (ed.), Marche, CNR, Comitato Nazionale per la Geografia, pp. 95-147.

CENCINI C. \& VARANI L. (1991), «Per una storia ambientale delle pianure costiere medioAdriatiche ", Geografia Fisica e Dinamica Quaternaria, 14, pp. 33-44.

CILLA G. \& DRAMIS F. (1999), « Aspetti del paesaggio nelle Marche centrali durante l'olocene Antico e Medio : l'evoluzione dei fondivalli ", in SILVESTRINI M. (ed.), Atti dell'Incontro di Studio « Recenti acquisizioni, problemi e prospettive della ricerca sull'Eneolitico dell'Italia Centrale ", Arcevia, 14-15 maggio 1999, Ministero per i Beni e le Attività Culturali, Soprintendenza Archeologica per le Marche \& Regione Marche - Centro Beni Culturali), pp. 73-101.

COLTORTI M. (1997), « Human impact in the Holocene fluvial and coastal evolution of the Marche region, Central Italy », Catena, 30, pp. 311-335.

COLTORTI M., NANNI T. \& VIVALDA P. (1991), « La bassa valle del fiume Musone (Marche) : geomorfologia e fattori antropici nell'evoluzione della pianura alluvionale », Geografia Fisica e Dinamica Quaternaria, 14, pp. 101-111.

CORREGIARI A., ROVERI M. \& TRINCARDI F. (1996), « Late Pleistocene and Holocene evolution of the North Adriatic Sea », Il Quaternario, 9, 2, pp. 697-704.

CURZI P.V. (1986), «Cenni di geologia dell'adriatico nel tratto marchigiano », Studi geologici camerti, volume speciale « La Geologia delle Marche », pp. 135-145.

DE BREUCK W. \& BEEUWSAERT E. (1997), « Geo-elektrische profileringsmethode voor het opsporen van archeologische resten », Natuurwetenschappelijk Tijdschrift, 76, Gent, pp. 3-8.

GORI U. (1988), « Contributo alla conoscenza della sedimentazione delle alluvioni quaternarie del fiume Foglia (Marche) », Geografia Fisica e Dinamica Quaternaria, 11, pp. 121-122.

HESSE A. (2000), «La mesure de la résistivité (ou de la conductivité) électrique du sol en prospection archéologique ", in PASQUINUCCI M. \& TRÉMENT F. (eds.), Non-destructive Techniques Applied to Landscape Archaeology, Oxbow Books, Oxford, pp. 104-113.

LEVEAU P., WALSCH K., TRÉMENT F. \& BARKER G. (1999), « Environmental reconstruction in Mediterranean landscape archaeology », in BARKER G. \& MATTINGLY D. (eds.), The archaeology of Mediterranean landscapes. Oxbow books, vol. 2, Oxford, 225 p. 
LUNI M. (1981), « Nuove tracce della frequentazione greca dell'Adriatico occidentale e riconoscimento dello scalo marittimo greco di S Marina di Focara (Pesaro) », Lincei Rendiconti Morali, Serie VIII, XXXVI, 1/2, pp. 45-78.

MARCHETTI M. (2002), « Environmental changes in the central Po Plain (northern Italy) due to fluvial modifications and anthropogenic activities », Geomorphology, 44, pp. 361-373.

MOSCATELLI U. \& VETTORAZZI L. (1988), « Aspetti delle divisioni agrarie romane nelle Marche », Le Marche - Archeologia, Storia, Territorio, Arcevia, pp. 7-84.

NANNI T. \& VIVALDA P. (1987), « Influenza della tettonica trasversale sulla morfogenesi delle pianure alluvionali marchigiani », Geografia Fisica e Dinamica Quaternaria, 10, pp. 180-192.

ORTOLANI M. \& ALFIERI N. (1947), « Deviazioni di fiumi piceni in epoca storica », Rivista Geografica Italiana, 54, pp. 2-16.

ORTOLANI M. \& ALFIERI N. (1978), « Sena gallica, in ALFIERI N. (ed.), Una città adriatica. Insediamento, forme urbane, economia e società nella storia di Senigallia, pp. 21-29.

PERCOSSI SERENELLI E. (ed.) (2001), Potentia : Quando poi scese il silenzio, Federico Motta Editore S.p.A., Milano, 189 p.

RENFREW C. \& BAHN P. (2000), Archaeology: Theories, Methods and Practice (Third edition), Thames \& Hudson, London, $640 \mathrm{p}$.

SERVIZIO GEOLOGICO D'ITALIA (1967), Carta geologica d'Italia 1 :100 000, F124 Macerata, Roma, Servizio geologico d'Ialia.

VAN DAMME D. (1984), « The freshwater Mollusca of Northern Africa: Distribution, Biogeography and Palaeoecology », Developments in Hydrobiology, 25, pp. 1-164, Junk, Den Haag.

VERHOEVEN G. \& DE VLIEGHER B.M. (2003), « Oblique Aerial Photography in a GIS Environment for Geo-Archaeological Research. A Case Study: The Potenza Valley Survey ", in GOOSSENS R. (ed.), Remote Sensing in Transition. Proceedings of the $23^{\text {rd }}$ Symposium of the European Association of Remote Sensing Laboratories, Ghent, Belgium, 2-5 June 2003, Rotterdam, pp. 427-434.

VERMEULEN F. \& DE DAPPER M. (eds.) (2000), Geoarchaeology of the landscapes of classical antiquity. Babesch - Bulletin antieke beschaving - Annual Papers on Classical Archaeology, Supplement 5, $233 \mathrm{p}$.

VERMEULEN F., MONSIEUR P. \& BOULLART C. (2002), « The Potenza Valley Survey: preliminary report on field campaign 2001 ", Babesch - Bulletin antieke beschaving - Annual Papers on Classical Archaeology, 77, pp. 49-71.

VERMEULEN F. \& VERHOEVEN G. (2004), « The contribution of aerial photography and field survey to the study of urbanization in the Potenza valley (Picenum) », Journal of Roman Archaeology, 17, pp. 57-82.

ZUFFA M. (1959), « Tracce di uno scalo marittimo greco a S. Marina di Focara (Pesaro) », Atti del I Convegno di Studi Etruschi, Studi Etruschi XXV, suppl., pp. 133-143.

\section{RÉSUMÉS}

Différentes techniques et méthodes des sciences de la Terre et de l'archéologie ont été combinées pour restituer l'évolution de la plaine côtière du fleuve Potenza, en Italie centrale, depuis l'Antiquité. Elles comprennent, d'une part, la prospection archéologique de surface et l'examen des photographies aériennes obliques de basse altitude pour déterminer l'occupation humaine et, 
d'autre part, les forages à la tarière et les sondages et profils géoélectriques afin de caractériser l'évolution de l'environnement naturel. La combinaison de ces méthodes avec l'analyse des sources écrites, l'examen des photographies aériennes verticales de haute altitude, les datations radiométriques ${ }^{14} \mathrm{C}$ et la détermination de mollusques permettent d'apporter de précieux éléments de réponses aux principales questions liées à l'histoire de la plaine côtière du fleuve Potenza. Ainsi les diverses positions du cours d'eau et du littoral depuis l'Antiquité ont été repérées.

We have combined geological, geomorphological and archaeological methods and techniques to reconstruct the evolution of the coastal plain of the Potenza river in central Italy since Antiquity. These methods and techniques comprise archaeological surface survey and the interpretation of oblique low-altitude aerial photographs to understand the evolution of human occupation on the one hand; hand augering and geoelectric survey to characterise the evolution of the natural environment on the other hand. The combination of these methods with archive study, interpretation of conventional vertical aerial photographs, radiocarbon dating, and analysis of molluscs have proved to be useful in resolving the main questions regarding the history of the coastal plain of the Potenza river. The different positions of the water course and of the coastline starting from Antiquity have been documented.

\section{INDEX}

Mots-clés : géoarchéologie, plaine côtière, prospection électrique de surface, Holocène, fleuve Potenza, les Marches, Italie

Keywords : geo-archaeology, coastal plain, geoelectric surface survey, Holocene, Potenza river, the Marches, Italy

\section{AUTEURS}

TANJA GOETHALS

Universiteit Gent, Tanja.Goethals@UGent.be

\section{MORGAN DE DAPPER}

Universiteit Gent, Morgan.DeDapper@UGent.be

FRANK VERMEULEN

Universiteit Gent, Frank.Vermeulen@UGent.be

\section{DIRK VAN DAMME}

Universiteit Gent, Dirk.VanDamme@UGent.be

KRISTINE WALRAEVENS

Universiteit Gent, Kristine.Walraevens@UGent.be 